\title{
ASSOCIATED CHEST INJURIES
}

By H. L. Frankel, M.B., M.R.C.P.

National Spinal Injuries Centre, Stoke Mandeville Hospital

INJURIES of the chest are common in association with traumatic paraplegia. In patients with dorsal lesions they are almost always present and in patients with dorso-lumbar lesions they are common. Fortunately, they are uncommon in patients with cervical lesions. They are relatively common in patients with gunshot wounds. The chest injuries have to be diagnosed and treated within the limitations set by the other injuries, which means, among other things, that chest X-rays have to be taken A.P. with the patient supine.

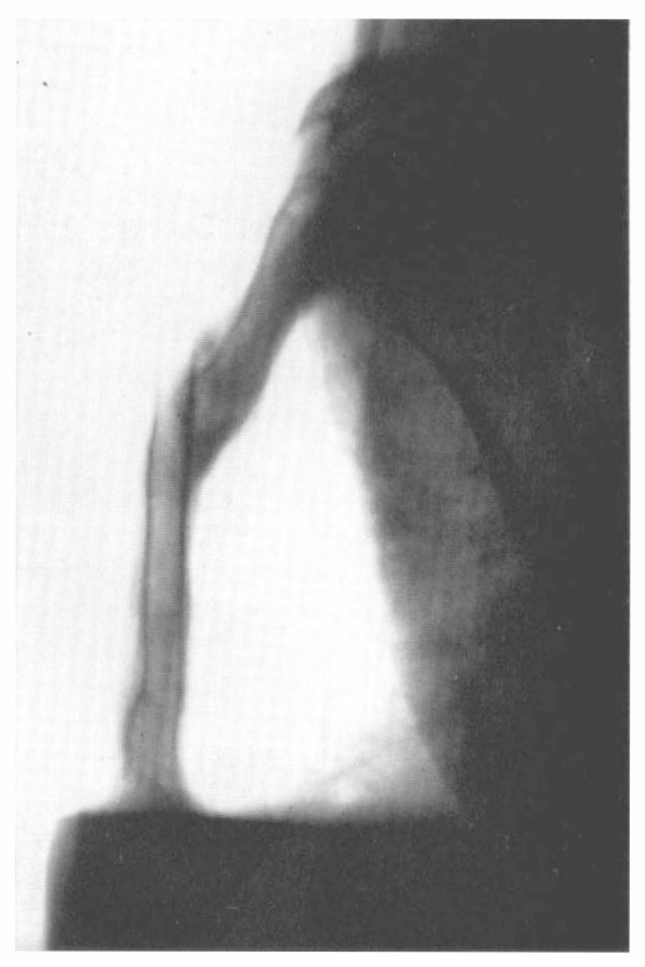

FIG. I

Fractured Ribs. Uncomplicated rib fractures cause pain on breathing and coughing, leading to retention of sputum which may cause bronchial obstruction and collapsed lung. Treatment is by manual support of the chest during coughing; less important but useful are broncho-dilator drugs, inhalations and antibiotics. Occasionally, the careful use of analgesics or local anaesthetics is justified.

Unfortunately, many of our patients have suffered from bronchitis before their injury and an even larger number have been heavy smokers. This greatly 
increases the complications after chest injuries. The main problem is clearing of sputum from the bronchial tree, and for this we rely heavily on our physiotherapists who are on duty day and night. In some cases, they give percussion and postural drainage and support the chest during coughing, but often, their most valuable contribution is in teaching the patient how to cough. An experienced physiotherapist can often get a patient with broken ribs to cough up his sputum without laying a hand on him. A good physiotherapist is worth more than all our drugs.

If many ribs are fractured, particularly if ribs are fractured at both ends and the sternum is fractured (fig. I), gross paradoxical movements or a 'flail chest' may develop. Not only are inspiration, expiration and coughing grossly inefficient, but the deformity often worsens in response to greater respiratory efforts. In addition, the movement of the broken ribs further endangers the underlying structures. We have treated several such patients with intermittent positive pressure respiration and this treatment has been spectacularly successful. As long as there is a genuine flail chest, the patients relax well and claim almost complete relief of dyspnoea and pain.

The use of the 'Stoke Mandeville-Egerton' tilting-turning bed (Guttmann, 1967) has made the management of patients with these gross chest wall injuries easier, safer and much more comfortable.

If fractured ribs or a spike of bone from a fractured vertebral body penetrate the pleura or lungs, a haemothorax and or a pneumothorax may develop.

Haemothorax. A haemothorax may develop slowly and not show on the first X-ray. Later dullness to percussion, a 'veil' over one side of the chest on $\mathrm{X}$-ray and dyspnoea may develop. If there is a moderate-sized bilateral haemothorax, it may be difficult to detect on an X-ray taken with the patient supine because the radiographer will develop the film until there is a reasonable and equal density to the lung fields. However, detection of the lung edge away from the chest wall will give a clue. If the haemothorax is not aspirated the patient may develop a persistent high temperature, even when the effusion is sterile. I do not know the mechanism of this fever which is associated with a high E.S.R. as some patients with equally large effusions following haemothorax have normal temperatures.

Haemothorax can usually be adequately treated by aspiration through a wide bore needle. It is difficult to aspirate a chest completely with the patient lying flat and a second aspiration a few days later is usually needed. Unless bleeding persists, I prefer to aspirate a simple haemothorax rather than put in an intercostal drain as I think the risk of infection is less.

Pneumothorax. I think that an intercostal drainage tube connected to an underwater seal should be used as soon as a traumatic pneumothorax is diagnosed. This condition is more dangerous than simple haemothorax and, compared with the risk of sudden increase in size and tension of the pneumothorax, the risks of tube drainage are slight.

Diaphragm. The diaphragm or phrenic nerve are sometimes injured. The diagnosis of a large tear of the diaphragm is usually made at laparotomy or at post-mortem as there is usually other intra-abdominal damage. Lesser damage 
is often suspected but rarely proved. Bowel sounds may be heard quite high in the chest with elevation of the diaphragm due to any cause. Diagnosis may depend on demonstrating a hollow viscus in the chest on X-ray either as a gas bubble or by using contrast media.

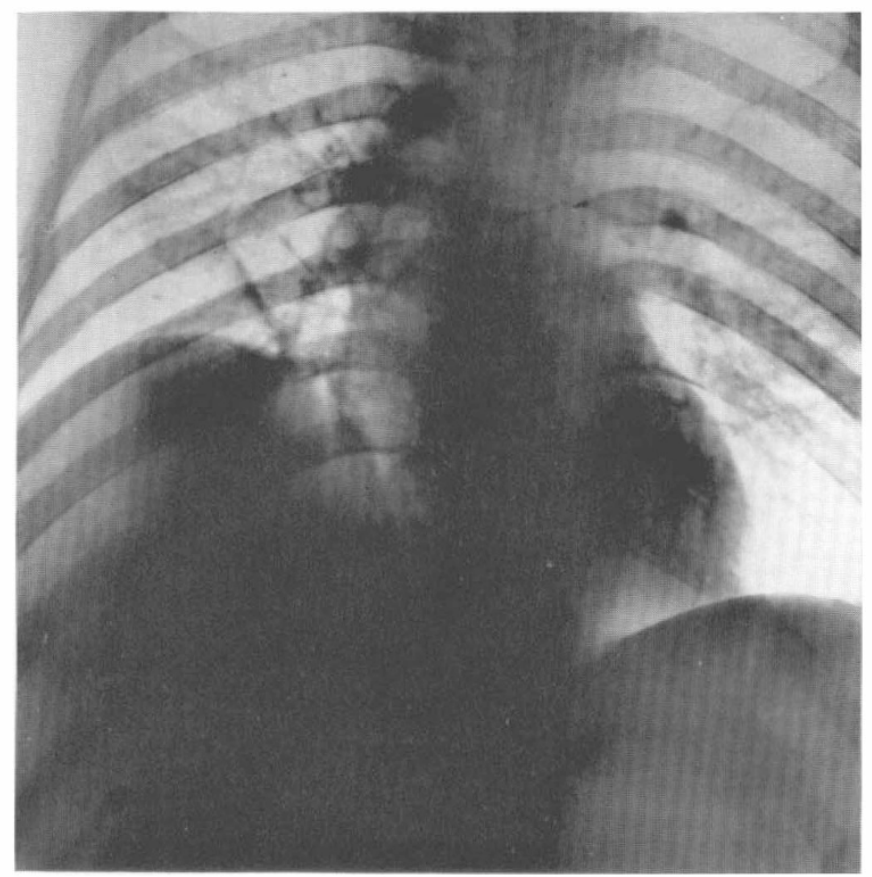

FIG. 2

The phrenic nerve is sometimes injured (fig. 2). This patient had a high diaphragm on admission. There was a history of inhalation of vomit and we suspected a blocked bronchus, but the diaphragm returned to its normal position in three months.

Lung Damage. Contusion of the lung parenchyma may give rise to what has been called 'traumatic pneumonopathy'. There is interstitial bleeding and oedema and blockage of alveolae and bronchioles with blood and exudate. There is no specific treatment. Oxygen, assisted coughing, bronchodilators, humidification and antibiotics have been used. Fortunately, large areas of lung are only rarely involved.

Pulmonary Vessels. I have not seen severe damage to the pulmonary vessels but we have had two patients with chest injuries who had a cerebral embolism. I believe these emboli may have originated in the pulmonary veins.

Heart and Mediastinum. The heart and mediastinal structures may be damaged. A 'bruised heart' may give clinical and electrocardiographic signs 
similar to a coronary thrombosis. Delayed rupture of the heart has been described by Tribe (I96I) and we have seen a case of delayed rupture of the aorta at postmortem (fig. 3). This man lived for several days after his accident.

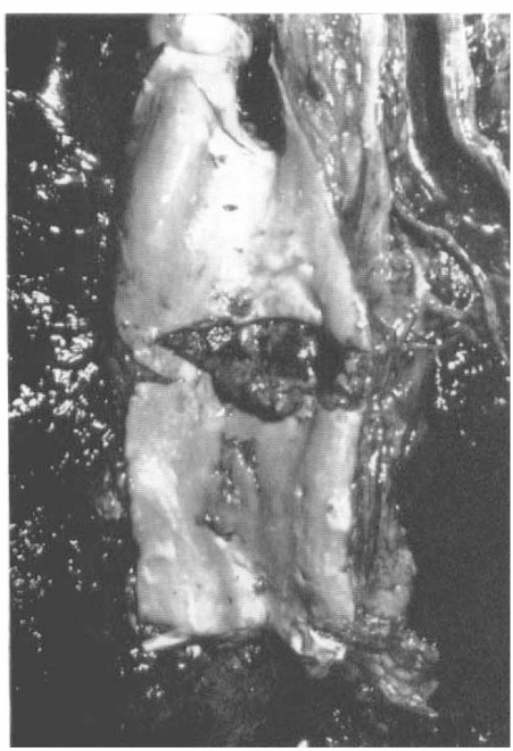

FIG. 3

Tracheostomy. Tracheostomy is often used in the management of chest injuries. I will not review the indications and complications except to say that our complication rate is roo per cent.-all the tracheas became infected. Crusting in the tube may occur (fig. 4). A patient with a high cervical cord lesion due to a gun-shot wound was transferred here from Aden by airplane on intermittent positive pressure respiration. On the journey his tracheostomy tube became displaced and on admission his face was expanding with each stroke of the respirator (fig. 5). He was cyanosed and deeply unconscious and as far as I could see was being oxygenated only through the subcutaneous tissues of his face and neck. With considerable difficulty we managed to place a tube back in his trachea and the surgical emphysema subsided in a week. Also Dollfus and I (1964) have described vagal cardiac arrest following intratracheal suction. In the last year, we have had two patients who developed a low tracheal stenosis. Both had been treated with intermittent positive pressure respiration and only plastic tubes had been used. The cuff had been let down at least two-hourly. The stenosis was difficult to recognise; in both cases it started with expiratory difficulty and the signs of bronchial asthma. By the time we recognised the stenosis, it had closed almost to a pin hole and we dilated the stenosis when the patients were in extremis and had lost consciousness. Both patients are still dependent on the use of long 'Chevalier Jackson' tracheostomy tubes which pass the stricture 
and hold it open. We had previously seen patients who developed stenosis above the stoma but these cases in which it occurred below the stoma are much more dangerous.

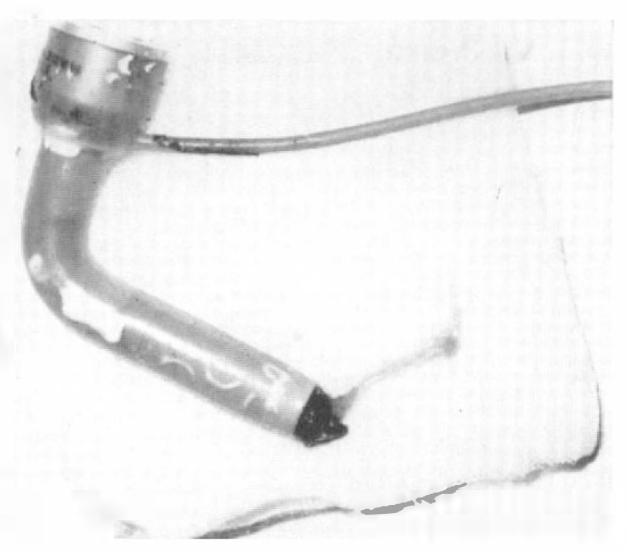

FIG. 4

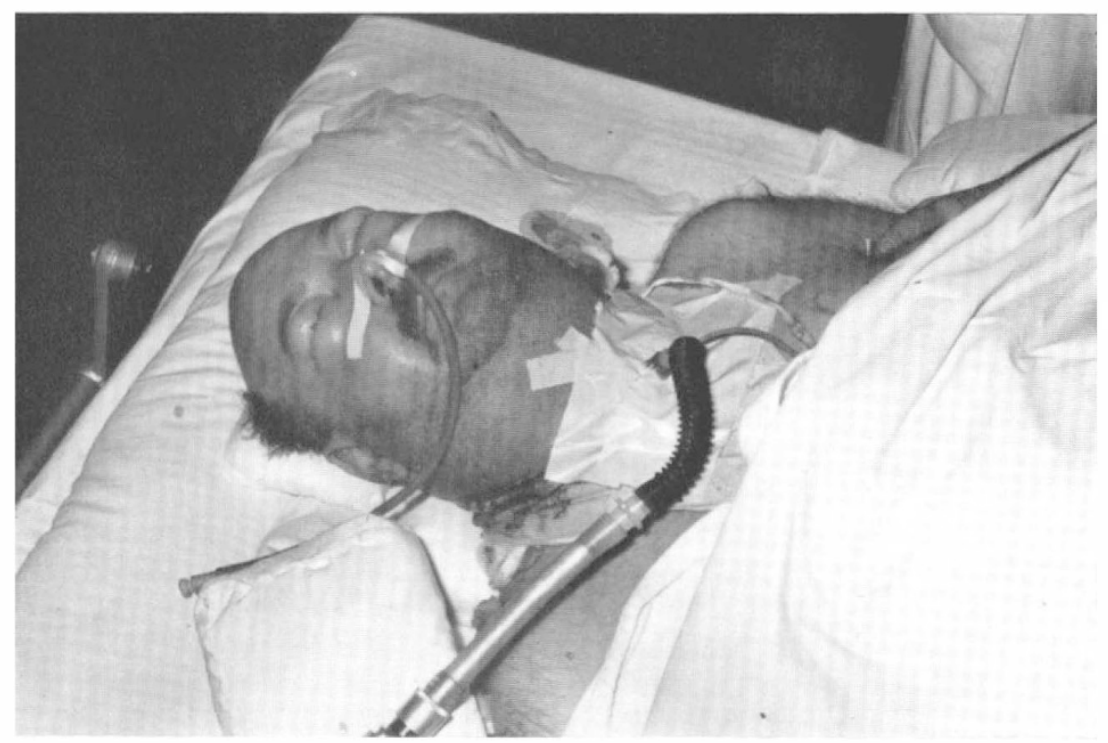

FIG. 5

Complications of tracheostomy are common and serious. We should reserve this operation for essential cases.

\section{REFERENCES}

Dollfus, P. \& Frankel, H. L. (1964). Int. F. Paraplegia, 2, 227.

GutTMANN, Sir L. (I967). Br.med.f., I, 288.

TRIBE, C. R. (I96I). Br. med. F. 2, I289. 